\title{
A Hybrid Modified Whale Optimization Algorithm with Simulated Annealing for Terrorism Prediction
}

\author{
Ghada M.A. Soliman ${ }^{*}$, Tarek H.M. Abou-El-Enien ${ }^{1}$, Eid Emary ${ }^{2}$, Motaz M.H. Khorshid ${ }^{1}$ \\ ${ }^{1}$ Faculty of Computers \& Artificial Intelligence, Cairo University, Giza, Egypt \\ ${ }^{2}$ Faculty of Computer Studies, Arab Open University, Cairo, Egypt
}

Corresponding Author Email: gh.tolan@ fci-cu.edu.eg

https://doi.org/10.18280/isi.240308

Received: 1 April 2019

Accepted: 7 June 2019

\section{Keywords:}

hybrid algorithms, memetic algorithm, whale optimization algorithm, feature selection, spiral path, tournament selection

\begin{abstract}
The main purpose of this research is to propose a novel hybrid memetic searching optimization algorithm, the proposed algorithm integrates the modified whale optimization algorithms with simulated annealing algorithm in a novel approach in order to enhance the searching capabilities of the modified whale optimization algorithm with the help of simulated annealing (SA). The proposed algorithm is used to find the minimum feature subset based on hybrid modified whale optimization algorithms and simulated annealing (WOA2SA, WOA3SA), where SA is embedded into the modified WO algorithms to achieve that good balance between (exploitation) and (exploration) capabilities of the modified algorithms. The resulting memetic algorithm improve the performance of the general classification tasks and hence had been used in the prediction of terrorist group (s) which responsible of the terror attacks on Egypt based on GTD terrorism data. The findings of this research can serve as an alarm tool to minimize the terrorist attacks on a certain region (country).
\end{abstract}

\section{INTRODUCTION}

In combinatorial optimization (NP-hard) problems; computing optimal solutions is computationally intractable, and so most researchers are usually satisfied with what known as "good" solutions that are obtained by metaheuristic algorithms. In addition to single solution search algorithms such as simulated annealing (SA) [1], tabu search [2], greedy heuristic [3], there is a growth interest in population based metaheuristics especially evolutionary algorithms (EA) such as genetic algorithms (GA) [4], genetic programming [5], ant colonies (AC) [6], scatter search (SS) [7], moth-flame optimization algorithm [8] and other numerous of new algorithms.

The hybridization of metaheuristics rises as a new research field in optimization due to the fact that the focus of research has shifted from an algorithm-oriented point of view to a problem-oriented point of view. The research in the last years have produced a large number of algorithms that simply do not fit into a single metaheuristic category. This is because these untraditional research approaches combine various algorithmic ideas, mostly originated from several branches of artificial intelligence, operations research and computer science. Such approaches are referred to as hybrid metaheuristics [9]. The hybrid algorithms have the ability for improving run time, results or both. Research on hybrid metaheuristics can be subdivided into five different categories, namely, the hybridization of metaheuristics with (meta-) heuristics which will be our main focus in this research. The other categories are constraint programming, tree search methods, problem relaxations, and dynamic programming.

The hybridization of metaheuristics with (meta-) heuristics is quite popular, especially whose concentrates on using of local search methods inside population-based methods; because the major strength of population-based methods in their exploration capability and the strength of local search methods in their rather fast intensification capability; so the hybrid algorithms can provide more efficient behavior and higher flexibility, in the field of evolutionary algorithms these hybrid algorithms are known as own name, memetic algorithms [10].

In this research; a novel hybrid memetic algorithm are presented that based on hybridization between the modified whale optimization algorithms (WOA2, WOA3) with the simulated annealing into a classification mechanism to provide a prediction system for one of the NP-hard real problems with high performance. The hybrid memetic algorithm proved its capability to enhance the classification performance and feature selection with highly accuracy and hence used in the prediction of the terrorist group(s) whose responsible of the terror attacks on Egypt.

The remainder of this paper is organized as follows: Section 2 presents an overview about the simulated annealing algorithm, while Section 3 explains in details the modified whale optimization algorithms. In Section 4; the proposed hybrid memetic algorithm components are introduced as well as the implementation, the results and analysis of the model are presented. Section 5 gives the main conclusion of the research and further points for future research.

\section{SIMULATED ANNEALING}

Simulated Annealing (SA) is a random and local search technique [11] was developed in order to solve the difficult and highly nonlinear optimization problems. SA is a robust and general techniques as it has the ability to deal with many constraints' problems, the noisy and chaotic data problems. SA 
based on using the highly similarity between the cooling and freezing way in which a metal cools and freezes into a minimum energy crystalline structure that is called as "The Annealing", and the search for a minimum solution in a more general system; SA is a basis that allow an optimization technique to solve the combinatorial problems as it can deal with other different disciplines problems. SA has a very good ability to escape from the local optima in which the improving move will be accepted but the worse one will be accepted as a solution under determined probability. The probability of accepting the worse solution is calculated by using the Boltzmann probability as follows in Eq. (1).

$$
\text { Probability }=\mathrm{e}^{-\propto / \mathrm{Temper}}
$$

where $\propto$ : objective difference evaluation between best solution $\left(\operatorname{Soln}_{\text {best }}\right)$ and the trail solution $\left(\operatorname{Soln}_{\text {trial }}\right)$, Temper: Temperature that periodically decreases during the search process.

And hence we can conclude that SA has main advantages over other local search algorithms in its high flexibility and the capability to approach global optimality as well as it does not depend on a restrictive structure that makes the algorithm in Figure 1 is quite versatile and can easily tuned to be used with other searching algorithms.

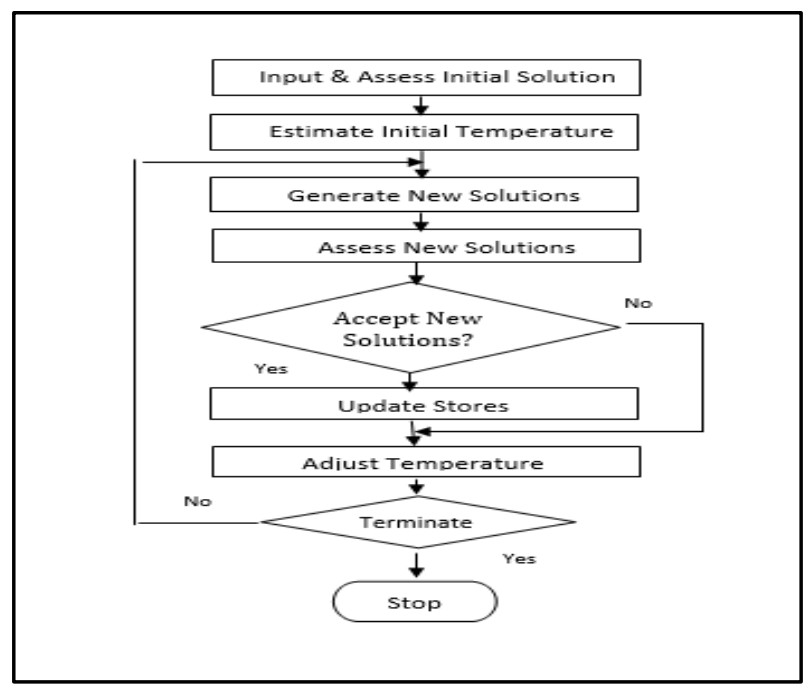

Figure 1. Simulated annealing algorithm

\section{MODIFIED WHALE ALGORITHMS (WOA2, WOA3) \\ OPTIMIZATION}

In Whale optimization algorithm [12]; the humpback whales which are special kind of whales hunt a school of krill or small fishes in which they are in a near area to the surface by creating a trap consists from bubbles called bubble-net strategy. The humpback whales have the ability to swim down in water in approximate of (10-15 meters) around those fishes with creating distinctive bubbles following a helix-shaped movement. The helix-9 shaped is defined as a spiral shape along a circle. Then the prey can follow the bubbles and moves upward the surface to be hunted by whales, as showed in Figure 2. The whales have different abilities allow it to be one of the promising algorithms and more powerful, efficient algorithm to lead to the best global search such as the ability to operate on a random basis or best agent while chasing the prey, as well as using the spiral helix shape movement in hunting. The spiral shaped path that explained in the original WO algorithm is follows a logarithmic shape also called (equiangular spiral) ) but the constant property of this spiral function is not realistic for the whales while its movement (the constant property in which the angle formed by the radial vector, this vector is formed from a line between any point "W" on the spiral toward the center of the spiral is constant ) which is not accurate to represent the whales movement in reality, and hence we intended in our research in choosing a more accurate and realistic spiral shape after investigate different forms of spiral shaped to simulate the helixmovement of the whales that enhance the exploitation capability of humpback whales in the hunting mechanism.

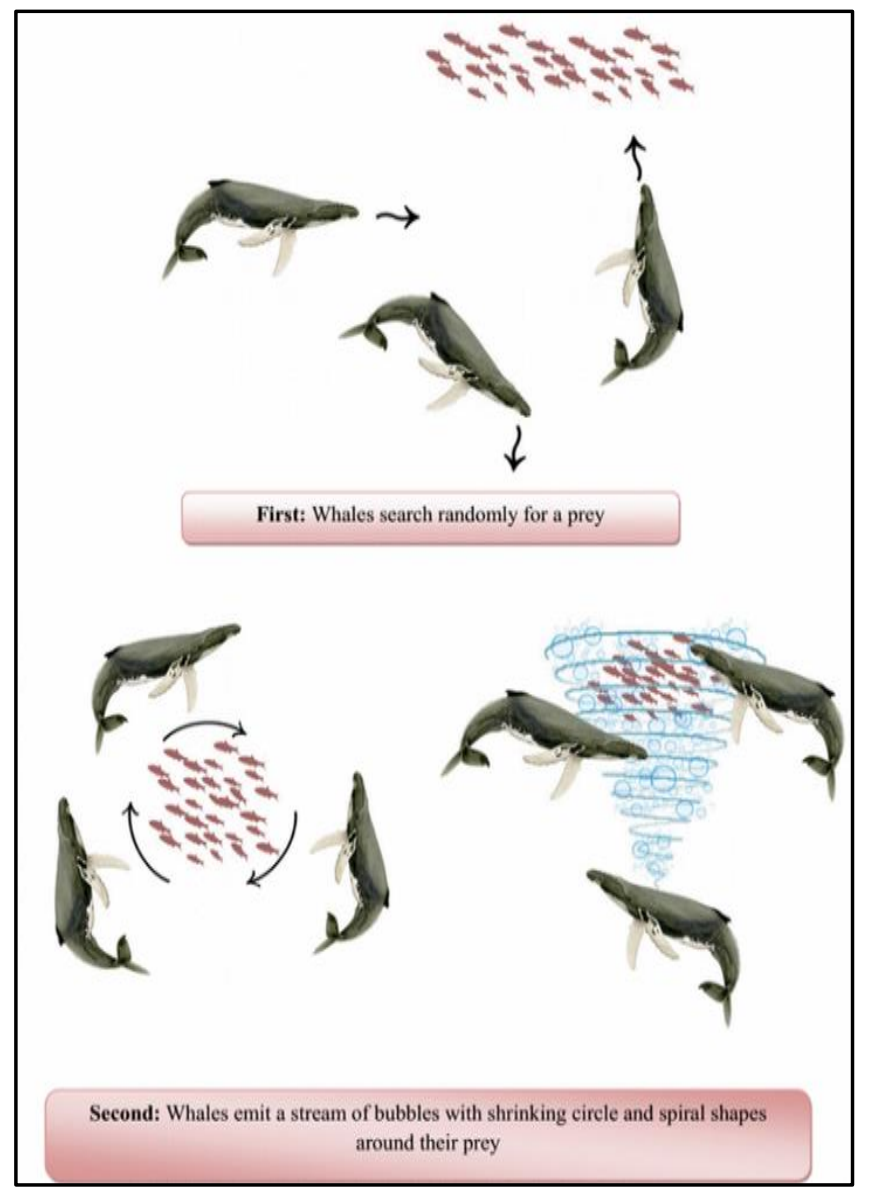

Figure 2. Bubble-Net haunting behavior of humpback whales

The new spiral shaped path that have been used in our research known as hyperbolic (reciprocal) and Archimedean spiral which proposed by Soliman et al. in [13] then investigate the performance of the modified versions of WO algorithm by conducting different experiments within a hybrid terrorism prediction system to predict the terrorist group(s) responsible of attacks on Egypt along the time period (from 1996 to 2017). The results of the modified WOA is tested, evaluated, then compared with the original algorithm, other two modified versions of the modified Moth flame optimization algorithm (MFO) and its modified versions (MFO2, MFO3) that have been proposed by Soliman et al in [14], and the popular algorithms such as Particle Swarm, and Genetic Algorithms. The main phases of WOA2, and WOA3; exploration and exploitation phases are presented below: 


\subsection{Exploitation phase}

\subsubsection{Shrinking encircling a prey}

The humpback whales begin to encircle the prey near the surface of the water as described mathematically by Eq. (2) and Eq. (3) and explained in $[9,12,13]$.

$$
\begin{aligned}
& \overrightarrow{\mathrm{W}}_{\mathrm{p}}(\mathrm{c}+1)=\overrightarrow{\mathrm{W}_{\mathrm{p}}{ }^{*}}(\mathrm{c})-\overrightarrow{\mathrm{M}} \cdot \overrightarrow{\mathrm{D}} \\
& \overrightarrow{\mathrm{D}}=\left|\overrightarrow{\mathrm{N}} \cdot \overrightarrow{\mathrm{W}_{\mathrm{P}}{ }^{*}}(\mathrm{n})-\overrightarrow{\mathrm{W}_{\mathrm{p}}}(\mathrm{c})\right| \\
& \overrightarrow{\mathrm{M}}=2 \overrightarrow{\mathrm{M}} \cdot \overrightarrow{\mathrm{s}}-\overrightarrow{\mathrm{M}} \\
& \overrightarrow{\mathrm{N}}=2 \overrightarrow{\mathrm{s}}
\end{aligned}
$$

where:

$\overrightarrow{\mathrm{W}_{\mathrm{p}}}$ : The whale's position vector.

$\overrightarrow{\mathrm{W}_{\mathrm{P}}{ }^{*}}$ : Represents historically best whales' position (solution) obtained so far.

c: indicates current iteration.

$\overrightarrow{\mathrm{M}}, \overrightarrow{\mathrm{N}}$ : known as coefficient vectors that are calculated as in Eq. (4) and Eq. (5) respectively.

$\overrightarrow{\mathrm{M}}$ : Called distance control parameter, its values is decreased from 2 till 0 within the iteration number.

$\vec{s}:$ A random vector follows the uniform distribution in the interval of $[0,1]$.

As explained by Eq. (2) the whales enhance their positions according to the position of the best known solution (optimum solution). Whales can be located in the neighborhood of small fishes via changing the values of $\vec{M}$ and $\vec{N}$ vectors; The Shrinking encircling behavior of a whale around the prey is achieved according to the decreasing value of $\overrightarrow{\mathrm{M}}$ in Eq. (4) that explained by Eq. (6)

$$
\overrightarrow{\mathrm{M}}=2-\mathrm{c} \frac{2}{\operatorname{MaxIn}}
$$

where

c: the number of the iteration.

MaxIn: maximum iterations.

\subsubsection{Bubble-net attacking method}

To determine the position of neighbor around the whale; the distance between a whale position $\left(\overrightarrow{\mathrm{W}_{\mathrm{P}}}\right)$ and the best known whale position obtained so far $\left(\overrightarrow{\mathrm{W}_{\mathrm{P}}^{*}}\right)$ has to be calculated. This distance is represented by spiral path called hyperbolic (reciprocal) spiral that we based on constructing the spiral path for our modified WO algorithm (WOA2) and Archimedes' Spiral that we based on in constructing the spiral function for (WOA3). These two spiral functions are illustrated and defined in [15].

\subsection{Mathematical formulation}

The Spiral paths equations that represent the humpback whales movement for WOA2 as explained by Eq. (7) and for WOA3 defined below in Eq. (8) as follows:

Hyperbolic Spiral:

$$
\overrightarrow{\mathrm{W}_{\mathrm{P}}}(\mathrm{c}+1)=\overrightarrow{\mathrm{D}} \cdot \frac{\cos (2 \pi \mathrm{r})}{\mathrm{r}}+\overrightarrow{\mathrm{W}_{\mathrm{P}}}(\mathrm{c})
$$

Archimedes' Spiral:

$$
\overrightarrow{\mathrm{W}_{\mathrm{P}}}(\mathrm{c}+1)=\overrightarrow{\mathrm{D}} \cdot \mathrm{r} \cos (2 \pi \mathrm{r})+\overrightarrow{\mathrm{W}_{\mathrm{P}}}(c)
$$

where

$\vec{D}=\left|\overrightarrow{\mathrm{W}_{\mathrm{P}}{ }^{*}}(\mathrm{c})-\overrightarrow{\mathrm{W}_{\mathrm{P}}}(c)\right|:$ is the distance do the whale number $\mathrm{j}$ - $t h$ whale to the prey (the last obtained best solution), and $r$ random number in $[-1,1]$.

The representation of the two hunting mechanisms of the whales (shrinking encircling and the bubble-net by spiralshape path), is by considering a $50 \%$ probability to make the choice between them in the optimization process as explained in the Eq. (9).

$$
\begin{aligned}
& \overrightarrow{\mathrm{W}_{\mathrm{P}}}(\mathrm{c}+1) \\
& =\left\{\begin{array}{cc}
\overrightarrow{W^{*}}(\mathrm{c})-\overrightarrow{\mathrm{M}} \cdot \overrightarrow{\mathrm{D}} & \text { if }(\mathrm{A}<0.5) \\
\overrightarrow{\mathrm{D}} \cdot \frac{\cos (2 \pi r)}{\mathrm{r}}+\overrightarrow{\mathrm{W}^{*}}(\mathrm{c}) & \text { if }(\mathrm{A} \geq 0.5) \\
\overrightarrow{\mathrm{D}} \cdot \mathrm{r} \cos (2 \pi r)+\overrightarrow{\mathrm{W}_{\mathrm{P}}}(\mathrm{c}) &
\end{array}\right.
\end{aligned}
$$

where: A considered a random number that defined in $[0,1]$.

\subsection{Exploration (diversification)}

The humpback whales can search for the prey via exploring the area globally and to enhance this exploration ability; they based on searching for the prey by a random selected whale to guide the search which is not depends on the whales' positions to each other. This mechanism is completely differs from the exploitation phase in which the whales can enhance and update their position depending on the best whales' position obtained so far, and hence, a vector $\mathrm{M}$ can take values "greater than 1" or "less than -1 " is used to allow the whales to move far away from the best whale position. The mathematical model for the exploration mechanism is as follows in Eq. (10) amd Eq. (11):

$$
\begin{gathered}
\overrightarrow{\mathrm{D}}=\left|\overrightarrow{\mathrm{N}} \cdot{\overrightarrow{\mathrm{W}_{\text {Prandom }}}}-\overrightarrow{\mathrm{W}_{\mathrm{P}}}\right| \\
\overrightarrow{\mathrm{W}}_{\mathrm{P}}(\mathrm{c}+1)=\overrightarrow{\mathrm{W}}_{\mathrm{P}_{\text {random }}}-\overrightarrow{\mathrm{M}} \cdot \overrightarrow{\mathrm{D}}
\end{gathered}
$$

where

$\overrightarrow{\mathrm{W}}_{\mathrm{P} \text { random }}$ : a random position vector represents a random chosen humpback whale.

The Following steps present the modified whale optimization algorithms (WOA2, WOA3). The algorithm begins with creating and initializing a set of humpback whales by using the uniform initialization method, then once the optimization starts; the position evaluation of these whales are done based on the defined fitness. The algorithm continue proceeding till finds the best whale position around the small fishes (the prey), the algorithm repeatedly executes the following steps until the maximum iteration reached.

Step 1: Update the main algorithm coefficients.

Step 2: a random value is generated that used to determine how the whales updates their position by using either one of the two mechanisms represented by Eq. (2) or Eq. (11) or based on the spiral paths explained in Eq. (7) and Eq. (8). 
Step 3: The solutions (whales' positions) cannot go outside the search space.

Step 4: The algorithm presents the output as the best whale's position with respect to the prey as an approximation to the global solution.

\section{PROPSED HYBRID MODIFIED WHALE OPTIMIZATION SIMULATED ANNEALING ALGORITHMS (WOA2SA, WOA3SA)}

This section presents the proposed hybrid metaheuristics algorithm between the Modified Whale optimization algorithms (WOA2, WOA3) with the Simulated Annealing (SA). The proposed hybrid model is used to find the minimum feature subset that used then to improve the performance of general classification tasks, and hence can perform the prediction of terrorist group (s) responsible of the terror attacks on Egypt. In the resulting memetic hybrid algorithm the SA algorithm is selected due to its high advantage in selection that embedded into (WOA2) and (WOA3) due to their ability in global search to achieve that good balance between (exploitation) and (exploration) abilities of the two algorithms.

The hybridization between the modified (WOA2 with SA) and (WOA3 with SA) based on using the "Tournament selection methodology" of SA algorithm instead of random selection mechanism in the exploration phase of Modified algorithms (WOA2) and (WOA3) as showed in Figure 3. The original WOA as well as the two modified versions (WOA2) and (WOA3) are using a blind operator to play the role of exploration or searching for the small fishes (prey), and hence we used the tournament selection operator in place of the random search which will be considered a solution as its initial state, and replace the original solution by the enhanced one.

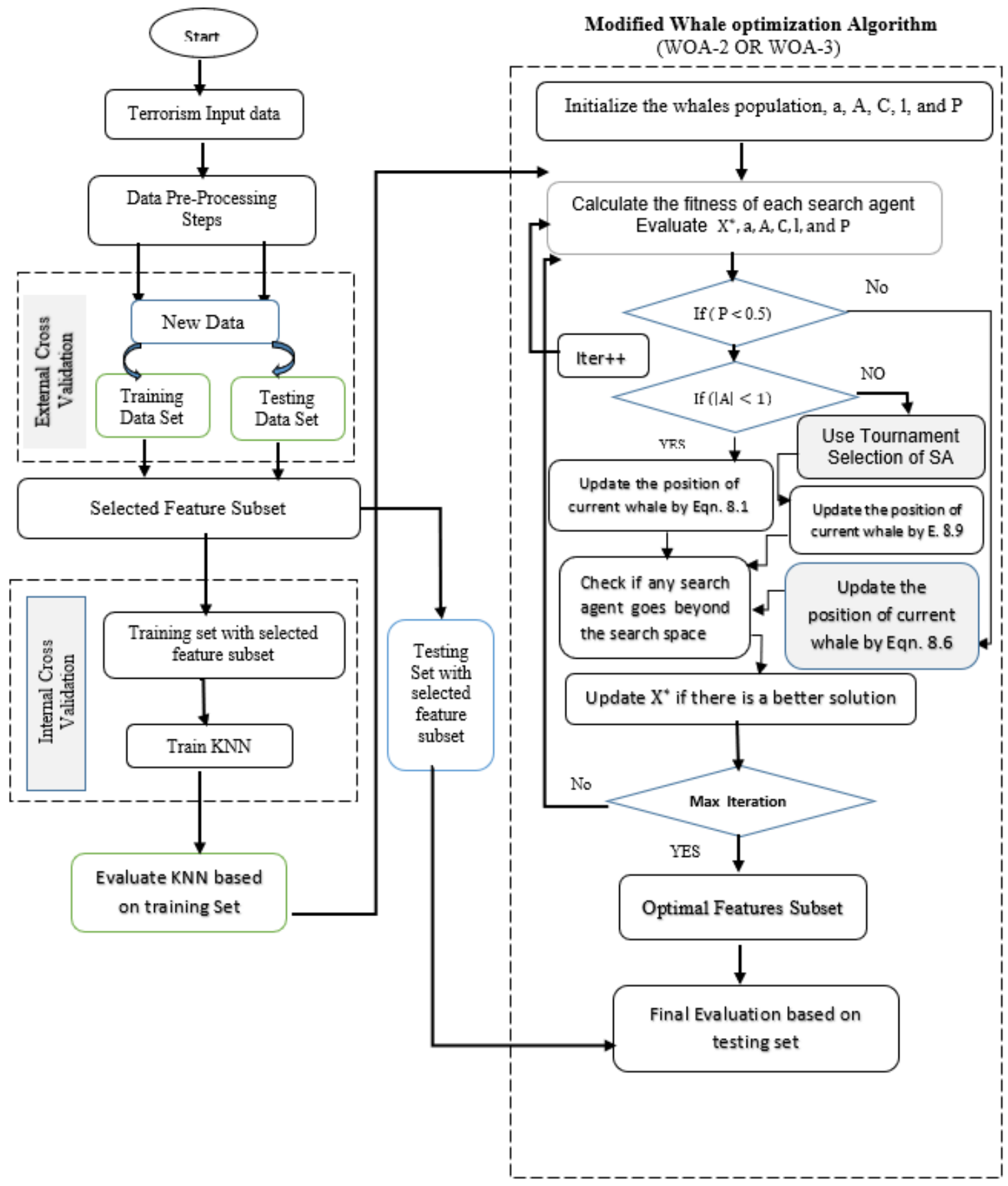

Figure 3. The hybrid memetic (WOA2SA) or (WOA3SA) algorithm 
Then the Eq. (10) and Eq. (11) will be replaced with the enhanced Eq. (12) and Eq. (13) as follows:

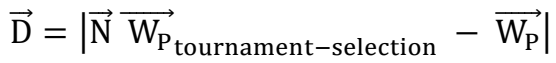

$$
\begin{aligned}
& \overrightarrow{W_{P}}(c+1)={\overrightarrow{W_{P}}}_{\text {tournament-selection }}-\vec{M} \cdot \vec{D}
\end{aligned}
$$

where

$\overrightarrow{\mathrm{W}}_{\mathrm{P}_{\text {tournament-selection }}}$ : is a position vector (represents the whale's position) chosen from the tournament selection process of the SA Algorithm.

\subsection{Data pre-processing}

This section explains how the terrorism data has been used and pre-processed in the proposed prediction model. The used data is real terrorism data and describes the terrorist attacks on Middle East \& North Africa especially the attacks that happened in Egypt (from year 1996 till year 2017) via the defined fitness function ACC which evaluate the accuracy of the model and explained in Eq. (14).

The data set derived from the Global Terrorism Database (GTD) which is taken from an open source of the National Consortium for the Study of Terrorism and Responses to Terrorism (START) [16]:

The data are required to be prepared for using in the classification process and it passed on multiple steps as explained below:

(1) Data Cleaning: Pre-process data in order to reduce noise and handle missing values by applying (Litwise-Deletion, Mode-Imputation) approaches.

(2) Data transformation (from Categorical into Numeric based on GTD coding system)

(3) The features in our data are classified as (Date, Incident location, Location Details, Attack Iinformation, Weapon Information, Target / Victim Information, Casualties \& Consequences) features

(4) For the time domain features; we applied a feature reduction where we transformed the "day, month, and yea" features into an equivalent "day per year, Hijiri day per year, and an equivalent day per week".

(5) For the position or location feature we transformed it into city-latitude, and city-longitude.

(6) Due to the huge number of classes in some features in our data; we had to combine the classes in some features into groups.

The terrorism data on the time period from year 1996 till year 2017 has 45 predictors (attributes or features) besides the feature "Terrorist group name" which is considered as (response class) that we should predict in our research; after data-preprocessing steps; the total number of attacks (records) became 813, the total set of features became 46 features as described below in Table 1 .

Table 1. Terrorism dataset features for the time period (1996 till 2017) of Egypt

\begin{tabular}{|c|c|c|c|}
\hline Feature & Type & Feature & Type \\
\hline Month & Numeric & Vicinity & Categorical \\
\hline Day & Numeric & Crit1 (Inclusion Criteria1) & Categorical \\
\hline Year & Numeric & Crit 2(Inclusion Criteria2) & Categorical \\
\hline Equivelant-Day Per Year & Numeric & Crit 3(Inclusion Criteria3) & Categorical \\
\hline Equivalent Hijiri day/year & Numeric & Doubtterr & Categorical \\
\hline Equivalent week per year & Numeric & Alternative & Categorical \\
\hline Equivalent day per week & Numeric & Multiple & Categorical \\
\hline Extended Incident & Categorical & Success) & Categorical \\
\hline Provstate & Text & Suicide & Categorical \\
\hline City & Text & Attack-Type & Categorical \\
\hline City-Latitude & Numeric & TargetType & Categorical \\
\hline City-Longitude & Numeric & Target-subtype & Categorical \\
\hline Specific & Categorical & Weapon-Type & Categorical \\
\hline Weapon-Subtype & Categorical & Compclaim & Categorical \\
\hline Nkill & Numeric & Property & Categorical \\
\hline Nkilter & Numeric & Propextent & Categorical \\
\hline Nwound & Numeric & Ishostkid & Categorical \\
\hline Nwoundte & Numeric & Nhostkid & Numeric \\
\hline Guncertain & Categorical & Nhours & Numeric \\
\hline Individual & Categorical & Ndays & Numeric \\
\hline Nperps & Numeric & Rnsome & Categorical \\
\hline nperpcap & Numeric & Ransomamt & Numeric \\
\hline Claimed & Categorical & Ransompaid & Numeric \\
\hline Claimmode & Categorical & Hostkidoutcom & Categorical \\
\hline Victim-Nationality & Categorical & Group-Name & Text \\
\hline
\end{tabular}

\subsection{Fitness function}

Fitness function is important for metaheuristic algorithms, it is used evaluate the quality of each subset. fitness evaluation is designed to combine the accuracy of classifier with the length of feature subset. A fitness function combines both the classification error (Classification accuracy) and the number of selected features and hence achieve that balance between 
the classification performance and the reduction size.

$$
\begin{aligned}
& \text { Maximize } \quad \text { ACC_Fitness }=\mathrm{CCR}(\mathrm{C}) \\
& \operatorname{CCR}(\mathrm{C})=\frac{N_{C}}{N}
\end{aligned}
$$

where

$\mathrm{CCR}(\mathrm{C})$ : is the classification performance measure that represents the correct classification ratio.

$\mathrm{N}_{\mathrm{C}}$ : is the number of correctly classified data instances and $\mathrm{N}$ : is the total number of instances in the data set.

\subsection{Results and analysis}

The implementation of the hybrid memetic algorithms WOASA, WOA2SA, WOA3SA are done using MATLAB R2015a, based on a set of assessment statistical indicators via different experiments as follows:

Table 2 presents the accuracy and mean fitness as well as the execution time for the memetic hybrid algorithms in different experiments with 5 searching agents (whales), in which we can observe that as increasing the number of iterations; the hybrid WOA2SA and WOA3SA perform better than the original WOA and better than the memetic (WOASA) in the accuracy of the algorithm as well as in the number of selected features.

Table 3 and Table 4 illustrate how the results are enhanced by increasing the number of search agents of the hybrid memetic algorithms where the mean fitness and the accuracy of the modified (WOA2), and (WOA3) with SA are better than the original WOA as well as the results of the hybrid algorithms WOA2SA and WOA3SA are better than WOASA and giving promising performance than WOA.

Table 2. The Results of the hybrid memetic algorithms with

\begin{tabular}{|c|c|c|c|c|c|}
\hline Iteration & Alg. & $\begin{array}{c}\text { Mean } \\
\text { Fitness }\end{array}$ & ACC. & $\begin{array}{c}\text { Number } \\
\text { of } \\
\text { Selected } \\
\text { features }\end{array}$ & $\begin{array}{c}\text { Exe. } \\
\text { Time } \\
\text { (Sec.) }\end{array}$ \\
\hline \multirow[t]{4}{*}{ 5o Iter. } & WOA & 0.272975 & 0.725 & 16 & 4 \\
\hline & WOASA & 0.255810 & 0.614 & 15 & 138 \\
\hline & WOA2SA & 0.274970 & 0.5484 & 16 & 142 \\
\hline & WOA3SA & 0.260257 & 0.5809 & 21 & 142 \\
\hline \multirow[t]{4}{*}{70 Iter. } & WOA & 0.283738 & 0.706 & 17 & 6 \\
\hline & WOASA & 0.238657 & 0.685 & 15 & 194 \\
\hline & WOA2SA & 0.248016 & 0.580 & 21 & 200 \\
\hline & WOA3SA & 0.253790 & 0.563 & 17 & 194 \\
\hline \multirow[t]{4}{*}{100 Iter. } & WOA & 0.275424 & 0.681 & 18 & 9 \\
\hline & WOASA & 0.279005 & 0.651 & 23 & 269 \\
\hline & WOA2SA & 0.278778 & 0.528 & 22 & 267 \\
\hline & WOA3SA & 0.253790 & 0.5630 & 17 & 194 \\
\hline \multirow[t]{4}{*}{150 Iter. } & WOA & 0.282550 & 0.718 & 11 & 12 \\
\hline & WOASA & 0.269197 & 0.580 & 18 & 396 \\
\hline & WOA2SA & 0.239716 & 0.645 & 15 & 466 \\
\hline & WOA3SA & 0.259016 & 0.539 & 18 & 470 \\
\hline
\end{tabular}
5 whales

\begin{tabular}{|c|c|c|c|c|c|}
\hline Iterations & Alg. & $\begin{array}{l}\text { Mean } \\
\text { Fitness }\end{array}$ & ACC. & $\begin{array}{c}\text { Number } \\
\text { of } \\
\text { Selected } \\
\text { features }\end{array}$ & $\begin{array}{l}\text { Exe. } \\
\text { Time } \\
\text { (Sec.) }\end{array}$ \\
\hline \multirow[t]{4}{*}{50 Iter. } & WOA & 0.309281 & 0.692 & 23 & 4 \\
\hline & WOASA & 0.287419 & 0.613 & 17 & 209 \\
\hline & WOA2SA & 0.290318 & 0.619 & 19 & 300 \\
\hline & WOA3SA & 0.294525 & 0.640 & 16 & 297 \\
\hline \multirow[t]{4}{*}{70 Iter. } & WOA & 0.323687 & 0.679 & 26 & 6 \\
\hline & WOASA & 0.271408 & 0.611 & 14 & 257 \\
\hline & WOA2SA & 0.292869 & 0.518 & 15 & 229 \\
\hline & WOA3SA & 0.288599 & 0.560 & 16 & 228 \\
\hline \multirow[t]{4}{*}{100 Iter. } & WOA & 0.277410 & 0.723 & 13 & 7 \\
\hline & WOASA & 0.275210 & 0.587 & 20 & 314 \\
\hline & WOA2SA & 0.273454 & 0.586 & 23 & 321 \\
\hline & WOA3SA & 0.277414 & 0.664 & 16 & 328 \\
\hline \multirow[t]{4}{*}{150 Iter. } & WOA & 0.265170 & 0.72 & 21 & 11 \\
\hline & WOASA & 0.270952 & 0.684 & 19 & 493 \\
\hline & WOA2SA & 0.263168 & 0.556 & 23 & 474 \\
\hline & WOA3SA & 0.280909 & 0.5286 & 15 & 468 \\
\hline
\end{tabular}

Table 3. The results of the memetic algorithms with 7 whales

\section{CONCLUSION AND FUTURE RESEARCH}

In our research paper we proposed a novel hybrid memetic searching optimization algorithm, the proposed algorithm integrates the modified whale optimization algorithms WOA2, and WOA3 with simulated annealing algorithm in a novel approach in order to enhance the searching capabilities of the modified whale optimization algorithm with the help of simulated annealing (SA).

The proposed hybrid memetic algorithm is used to find the minimum feature subset with highest accuracy and maximum fitness based on hybrid modified whale optimization algorithms and simulated annealing (WOA2SA, WOA3SA), where SA is embedded into the modified whale optimization algorithms to achieve that good balance between (exploitation) and (exploration) capabilities of the modified algorithms. The hybrid memetic algorithms are employed to improve the performance of general classification tasks and so allow the hybrid memetic algorithm to predict the terrorist group (s) responsible of the terror attacks on Egypt during the time period from year 1996 to year 2017 based on the data derived from the Global Terrorism Database (GTD). The results of the novel memetic hybrid algorithms (WOA2SA), and (WOA3SA) proved the superiority in achieving higher accuracy and maximum fitness than the hybrid original algorithm WOASA as well as the single WOA which improve the prediction of the terrorist group(s) responsible of terrorist attacks on Egypt.

For future research, the proposed algorithm can be used to predict the terrorist groups (s) on different regions (countries), a researcher can also use a hybrid embeded features selection approach instead of wrapper approach that has been used in our research. A researcher can used different ensemble classification algorithms instead of KNN such as Random forests classification algorithm.

Another interesting research point; a hybridization between more than two metaheuristics could be applied in our proposed 
system such as a hybridization between a WOA2 or WOA3 with the Particle Swarm Optimization algorithm (PSO) and SA.

Table 4. The results of the memetic algorithms with 10 whales

\begin{tabular}{|c|c|c|c|c|c|}
\hline Iteration & Alg. & $\begin{array}{c}\text { Mean } \\
\text { Fitness }\end{array}$ & ACC. & $\begin{array}{c}\text { Number } \\
\text { of } \\
\text { Selected } \\
\text { features }\end{array}$ & $\begin{array}{c}\text { Exe. } \\
\text { Time } \\
\text { (Sec.) }\end{array}$ \\
\hline \multirow[t]{4}{*}{$\begin{array}{l}50 \\
\text { iterations }\end{array}$} & WOA & 0.30643 & 0.694 & 17 & 2.7 \\
\hline & WOASA & 0.30135 & 0.575 & 17 & 137 \\
\hline & WOA2SA & 0.28843 & 0.527 & 23 & 143 \\
\hline & WOA3SA & 0.29472 & 0.511 & 20 & 152 \\
\hline \multicolumn{6}{|l|}{$\begin{array}{l}\mathbf{7 0} \\
\text { iterations }\end{array}$} \\
\hline & WOA & 0.28374 & 0.715 & 24 & 4 \\
\hline & WOASA & 0.28852 & 0.591 & 14 & 230 \\
\hline & WOA2SA & 0.275 & 0.556 & 22 & 281 \\
\hline & WOA3SA & 0.29582 & 0.605 & 23 & 382 \\
\hline \multicolumn{6}{|l|}{$\begin{array}{l}100 \\
\text { iterations }\end{array}$} \\
\hline & WOA & 0.29404 & 0.707 & 17 & 8 \\
\hline & WOASA & 0.27411 & 0.605 & 21 & 353 \\
\hline & WOA2SA & 0.26309 & 0.527 & 18 & 363 \\
\hline & WOA3SA & 0.27149 & 0.621 & 23 & 332 \\
\hline \multicolumn{6}{|l|}{$\begin{array}{l}150 \\
\text { iterations }\end{array}$} \\
\hline & WOA & 0.29693 & 0.704 & 19 & 8 \\
\hline & WOASA & 0.2692 & 0.595 & 15 & 823 \\
\hline & WOA2SA & 0.29693 & 0.625 & 19 & 775 \\
\hline & WOA3SA & 0.28821 & 0.538 & 19 & 449 \\
\hline
\end{tabular}

\section{REFERENCES}

[1] Kirkpatrick, S., Gelatt, C.D., Vecchi, M.P. (1983). Optimization by simulated annealing. Science, 220(4598): $671-680$. https://doi:10.1126/science.220.4598.671

[2] Glover, F. (1989). Tabu Search-Part I. ORSA Journal of Computing, 1(3): 190-206. https://doi.org/10.1287/ijoc.1.3.190

[3] Lawler, E.L. (1976). Combinatorial Optimization: Networks and Matroids. Holt, Rinehart and Winston, USA.
[4] Holland, J.H. (1975). Adaptation in Natural and Artificial Systems. Ann Arbor, MI, USA: Michigan Press University.

[5] Koza, J.R. (1992). Genetic Programming. Cambridge, USA: MIT Press.

[6] Colorni, A., Dorigo, M., Maniezzo, V. (1991). Distributed optimization by ant colonies. In European Conf. on Artificial Life. Elsevier Publishing, pp. 134142.

[7] Glover, F. (1977). Heuristics for integer programming using surrogate constraints. Decision Sciences, 8: 156166. https://doi: 10.1111/j.1540-5915.1977.tb01074.x

[8] Mirjalili, S. (2015). Moth-flame optimization algorithm: a novel nature-inspired heuristic paradigm. Elsevier, Knowledge-based Systems, 89: 228-249. https://doi.org/10.1016/j.knosys.2015.07.006

[9] Blum, C., Blesa Aguilera, M.J., Roli, A., Sampels, M. (2008). Hybrid meta-heuristics - an emerging approach to optimization. Volume 114 of Studies in Computational Intelligence. Springer.

[10] Krasnogor, N., Smith, J. (2005). A tutorial for competent memetic algorithms: Model, taxonomy, and design issues. IEEE Transactions on Evolutionary Computation, 9(5): 474488. https://doi:10.1109/TEVC.2005.85020

[11] Ingber, L. (1993). Simulated annealing: Practice versus theory. Mathl. Comput. Modelling, 18(11): 29-57. https:// doi: 10.1016/0895-7177(93)90204-C

[12] Mirjalili, S., Lewis, A. (2016). The whale optimization algorithm. Advances in Engineering Software, 95: 51-67. https://doi.org/10.1016/j.advengsoft.2016.01.008

[13] Soliman, G.M.A., Khorshid, M., Abou-El-Enien, T.H.M. (2016). Modified moth-flame optimization algorithms for terrorism prediction. International Journal of Application or Innovation in Engineering \& Management, 5(7): 47-596.

[14] Soliman, G.M.A., Abou-El-Enien, T.H.M., Emary, E., Khorshid, M. (2018). A hybrid whale optimization algorithm with adaptive spiral for terrorism prediction (the case of Egypt). European Journal of Scientific Research, 149(2): 165-184.

[15] Bowser, E.A. (1880). An Elementary Treatise on Analytic Geometry: Embracing Plane Geometry and an Introduction to Geometry of Three Dimensions (4th ed.). New York, D. Van Nostrand.

[16] Global Terrorism Database (GTD). (2009-2018). University of Maryland. National Consortium for the Study of Terrorism and Responses to Terrorism. A Center of Excellence of the U.S. Department of Homeland Security. University of Maryland, USA. 